\title{
Theoretical Analysis and Atomistic Modelling of Diffusion and Stability of Pure Element Hollow Nanospheres and Nanotubes
}

\author{
A.V. Evteev ${ }^{1, a}$, E.V. Levchenko ${ }^{1, b}$, I.V. Belova ${ }^{1, c}$ and G.E. Murch ${ }^{1, d}$ \\ ${ }^{1}$ Diffusion in Solids Group, School of Engineering, \\ The University of Newcastle, Callaghan, NSW 2308, Australia

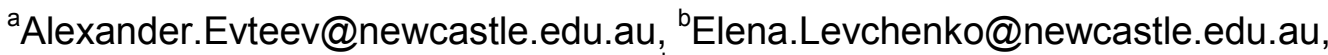 \\ Irina.Belova@newcastle.edu.au, dGraeme.Murch@newcastle.edu.au
}

Keywords: Diffusion, stability, defects, atomistic modelling, kinetic Monte Carlo, molecular dynamics, embedded atom method, hollow nanospheres, nanotubes.

\begin{abstract}
A theoretical and atomistic study of diffusion and stability of a pure element hollow nanosphere and nanotube is performed. The shrinkage via the vacancy mechanism of these hollow nano-objects is described analytically. Using Gibbs-Thomson boundary conditions an exact solution of the kinetic equation in quasi steady-state at the linear approximation is obtained. The collapse time as a function of the geometrical sizes of the hollow nano-objects is determined. Kinetic Monte Carlo simulation of the shrinkage of these nano-objects is performed: it confirms the predictions of the analytical analysis. Next, molecular dynamics simulation in combination with the embedded atom method is used to investigate diffusion by the vacancy mechanism in a Pd hollow nanosphere and nanotube. It is found that the diffusion coefficient in a Pd hollow nanosphere and nanotube is larger near the inner and external surfaces compared with the middle part of a nanoshell. The molecular dynamics results provide quite a strong but indirect argument that a real pure element hollow nanosphere and nanotube may not shrink as readily via the vacancy mechanism as compared with the predictions of the analytical analysis and kinetic Monte Carlo simulations.
\end{abstract}

\section{Introduction}

In recent years, the synthesis of uniform hollow nano-objects with easily tailored void sizes is of interest due to a wide range of technological applications such as catalysis, drug delivery vehicles, containment of environmentally sensitive species, components for highly sensitive colorimetric sensors, device components and many others [1,2]. Recently Sun et al. [1] have developed a simple and generic approach to the large-scale synthesis of hollow nanostructures made of $\mathrm{Au}, \mathrm{Pt}$ and $\mathrm{Pd}$, whose morphology, void size and wall thickness were all controlled by silver nanostructures that served as templates. The key step of this process is the redox reaction between a $\mathrm{Ag}$ template and the solution of the appropriate salt precursor. This approach has been successfully used to prepare hollow nanostructures of noble metals with a range of different morphologies such as spheres, tubes, triangular rings, prisms and cubes.

However, it has been noted $[3,4]$ that, in particular, hollow nanospheres should be unstable in principle and, with time, will have a tendency to shrink into a solid nanosphere. This is because the resulting reduction in surface area should be more advantageous from an energetic point of view: $\gamma\left(r_{\mathrm{i}}^{2}+r_{\mathrm{e}}^{2}\right)>\gamma r_{\mathrm{f}}^{2}\left(\gamma\right.$ is the surface energy, $r_{\mathrm{i}}$ and $r_{\mathrm{e}}$ are the inner and external radii of a hollow nanosphere, $r_{\mathrm{f}}$ is the radius of a collapsed compact nanosphere). In accordance with [3,4] the mechanism of shrinking can be considered as a result of the vacancy flux from the inner surface to the external surface. The driving force for such a flux is the difference between the vacancy concentrations $c_{\mathrm{V}}$ on the inner and external surfaces. In [3,4] only an analytical estimate of the collapse time of a pure element hollow nanosphere for the special case $r_{\mathrm{e}}-r_{\mathrm{i}}<<r_{\mathrm{i}}$ was obtained.

In this paper, using Gibbs-Thomson boundary conditions in quasi steady-state at the linear approximation, we first analytically describe shrinking via the vacancy mechanism of a pure element hollow nanosphere and nanotube, and determine the collapse time as a function of geometric size of the nano-objects. Next, we apply kinetic Monte Carlo (KMC) simulation to test the analytical analysis. Finally, we study the diffusion in a pure Pd hollow nanosphere and nanotube by performing a molecular dynamics (MD) simulation using the embedded atom method (EAM). 


\section{Phenomenological Analysis}

From the macroscopic thermodynamic point of view for a hollow nanosphere and nanotube with internal radius $r_{\mathrm{i}}$ and external radius $r_{\mathrm{e}}$, the variation of vacancy composition near the surfaces with the number of vacancies in the void $N_{\mathrm{V}}^{\mathrm{void}}$ is simply given by the Gibbs-Thomson equation:

$$
c_{\mathrm{V}}^{ \pm}=c_{\mathrm{V}}^{0} \exp \left( \pm \frac{\partial \Delta G_{\mathrm{S}} / \partial N_{\mathrm{V}}^{\mathrm{void}}}{k T}\right)
$$

where $c_{\mathrm{V}}^{0}$ is the equilibrium vacancy concentration near a planar surface, $\Delta G_{\mathrm{S}}=\gamma S$ is the free energy of the spherical or cylindrical surface with a principal radius of curvature $r\left(r=r_{\mathrm{i}}\right.$ or $\left.r=r_{\mathrm{e}}\right), S$ is the surface area and $\gamma$ is the surface energy per unit area. In the above formula, the curvature is taken as positive for the inner surface and negative for the external surface. Since for a spherical and cylindrical geometry we have that:

$$
\frac{\partial \Delta G_{\mathrm{S}}}{\partial N_{\mathrm{V}}^{\text {void }}}=\frac{\partial \Delta G_{\mathrm{S}}}{\partial r} \frac{\partial r}{\partial N_{\mathrm{V}}^{\text {void }}}=\frac{2 \gamma \Omega}{r} \text { and } \frac{\gamma \Omega}{r},
$$

respectively, Eq. 1 is reduced to:

$$
c_{\mathrm{V}}^{ \pm}=c_{\mathrm{V}}^{0} \exp \left(\frac{\beta}{ \pm r}\right)
$$

where $\beta=\beta_{\mathrm{s}}=2 \gamma \Omega / k T$ for a hollow nanosphere and $\beta=\beta_{\mathrm{c}}=\gamma \Omega / k T$ for a nanotube, and $\Omega$ is the atomic volume. In the following, we will assume that the equilibrium vacancy concentration $c_{\mathrm{V}}^{\mathrm{eq}}$ in the bulk of a system does not strongly differ from the equilibrium vacancy concentration near a planar surface $\left(c_{\mathrm{V}}^{0} \approx c_{\mathrm{V}}^{\text {eq }}\right)$ and, thereafter, we will use $c_{\mathrm{V}}^{\text {eq }}$ instead of $c_{\mathrm{V}}^{0}$. If the ratio $\beta / r<<1$, then, for the vacancy concentrations at the inner and the external surfaces of the hollow nano-objects a linear approximation [3-6] can be used:

$$
c_{\mathrm{V}}^{\mathrm{i}}=c_{\mathrm{V}}\left(r_{\mathrm{i}}\right)=c_{\mathrm{V}}^{\mathrm{eq}}\left(1+\frac{\beta}{r_{\mathrm{i}}}\right), \quad c_{\mathrm{V}}^{\mathrm{e}}=c_{\mathrm{V}}\left(r_{\mathrm{e}}\right)=c_{\mathrm{V}}^{\mathrm{eq}}\left(1-\frac{\beta}{r_{\mathrm{e}}}\right) .
$$

A linear approximation (Eqs. 4) is satisfactory (with a maximum error of 5\%) when $r_{\mathrm{i}}>3 \beta$.

The diffusion equations in spherical and cylindrical coordinates for the steady-state approximation of the vacancy concentration are [7]:

$$
\frac{\partial}{\partial r}\left(r^{2} \frac{\partial c_{\mathrm{V}}}{\partial r}\right)=0, \quad \frac{\partial}{\partial r}\left(r \frac{\partial c_{\mathrm{V}}}{\partial r}\right)=0
$$

respectively. With the boundary conditions of Eqs. 4 they have the following solutions:

$$
c_{\mathrm{V}}(r)=c_{\mathrm{V}}^{\mathrm{eq}}\left[1+\beta \frac{r_{\mathrm{e}}+r_{\mathrm{i}}-2 r}{r\left(r_{\mathrm{e}}-r_{\mathrm{i}}\right)}\right], \quad c_{\mathrm{V}}(r)=c_{\mathrm{V}}^{\mathrm{eq}}\left[1+\beta \frac{r_{\mathrm{e}} \ln \left(r_{e}\right)+r_{\mathrm{i}} \ln \left(r_{i}\right)-\left(r_{\mathrm{e}}+r_{\mathrm{i}}\right) \ln (r)}{r_{\mathrm{e}} r_{\mathrm{i}} \ln \left(r_{\mathrm{e}} / r_{\mathrm{i}}\right)}\right] .
$$

Then, according to Fick's First Law, the vacancy flux $J_{\mathrm{V}}$ from the void to the shell across the inner surface is:

$$
\Omega J_{\mathrm{V}}\left(r_{\mathrm{i}}\right)=-\left.D_{\mathrm{V}} \frac{\partial c_{\mathrm{V}}}{\partial r}\right|_{r=r_{\mathrm{i}}},
$$

where $D_{\mathrm{V}}$ is the diffusion coefficient of the vacancies. $D_{\mathrm{V}}$ is related to the measurable tracer diffusion coefficient by $D^{*}=c_{\mathrm{V}}^{\mathrm{eq}} D_{\mathrm{V}} f_{0}$ where $f_{0}$ is the geometric tracer correlation factor for the lattice. The radius $r_{\mathrm{i}}$ of the inner surface varies with time as:

$$
\frac{d r_{\mathrm{i}}}{d t}=-\Omega J_{\mathrm{V}}\left(r_{\mathrm{i}}\right)=-\frac{c_{\mathrm{V}}^{\mathrm{eq}} D_{V} \beta}{r_{\mathrm{i}}^{2}} \frac{r_{\mathrm{e}}+r_{\mathrm{i}}}{r_{\mathrm{e}}-r_{\mathrm{i}}} \quad \text { and } \quad \frac{d r_{\mathrm{i}}}{d t}=-c_{\mathrm{V}}^{\mathrm{eq}} D_{\mathrm{V}} \beta \frac{r_{\mathrm{e}}+r_{\mathrm{i}}}{r_{\mathrm{i}}^{2} r_{\mathrm{e}} \ln \left(r_{\mathrm{e}} / r_{\mathrm{i}}\right)}
$$

for a hollow nanosphere and nanotube, respectively. The external radius of the hollow nano-objects $r_{\mathrm{e}}$ changes can be found from the conditions of conservation of matter: 


$$
r_{\mathrm{f}}^{q}=r_{\mathrm{e}}^{q}-r_{\mathrm{i}}^{q}=\mathrm{const},
$$

where $q=3$ for a hollow nanosphere and $q=2$ for a nanotube. In order to obtain the analytical (closed form) solution of Eqs. 8 and 9, we introduce new non-dimensional variables and, making use of Eq. 9, we relate them to the old variables $[5,6]$ :

$$
\varepsilon=\frac{r_{\mathrm{i}}}{r_{\mathrm{e}}}, \quad \tau=\frac{6 c_{\mathrm{V}}^{\mathrm{eq}} D_{\mathrm{v}} t \beta}{r_{\mathrm{f}}^{3}}, \quad r_{\mathrm{i}}=\frac{\varepsilon r_{\mathrm{f}}}{\left(1-\varepsilon^{q}\right)^{\frac{1}{q}}}, \quad r_{\mathrm{e}}=\frac{r_{\mathrm{f}}}{\left(1-\varepsilon^{q}\right)^{\frac{1}{q}}} .
$$

Then Eqs. 8 can be rewritten as:

$$
\frac{d \varepsilon}{d \tau}=-\frac{1}{6} \frac{\left(1-\varepsilon^{3}\right)^{2}}{\varepsilon^{2}} \frac{1+\varepsilon}{1-\varepsilon}, \quad \frac{d \varepsilon}{d \tau}=\frac{1}{6} \frac{\left(1-\varepsilon^{2}\right)^{5 / 2}}{\varepsilon^{2}} \frac{1+\varepsilon}{\ln (\varepsilon)}
$$

for a hollow nanosphere and nanotube respectively. These equations can be readily solved analytically to give the solutions (where we defined $\delta=\varepsilon / \varepsilon_{0}$ and $\varepsilon_{0}=r_{\mathrm{i} 0} / r_{\mathrm{e} 0}$ is the ratio of the inner $r_{\mathrm{i} 0}$ and the external $r_{\mathrm{e} 0}$ radii of the hollow nano-objects at the initial point of time $t=0$ ):

$$
\tau(\delta)=\ln \left[\frac{\left(1-\varepsilon_{0}\right)\left(1+\varepsilon_{0}\right)^{3}}{\left(1-\varepsilon_{0}^{3}\right)^{\frac{4}{3}}} \frac{\left(1-\varepsilon_{0}^{3} \delta^{3}\right)^{\frac{4}{3}}}{\left(1-\varepsilon_{0} \delta\right)\left(1+\varepsilon_{0} \delta\right)^{3}}\right]-2 \varepsilon_{0}\left(\frac{1-\varepsilon_{0}}{1-\varepsilon_{0}^{3}}-\delta \frac{1-\varepsilon_{0} \delta}{1-\varepsilon_{0}^{3} \delta^{3}}\right)
$$

for hollow nanosphere [5] and

$$
\begin{aligned}
\frac{5}{4} \tau(\delta)= & \ln \left[\frac{1+\left(1-\varepsilon_{0}^{2} \delta^{2}\right)^{\frac{1}{2}}}{1+\left(1-\varepsilon_{0}^{2}\right)^{\frac{1}{2}}}\right]+\arcsin \left(\varepsilon_{0} \delta\right)-\arcsin \left(\varepsilon_{0}\right)+\varphi_{1}\left(\varepsilon_{0}\right)-\varphi_{1}\left(\varepsilon_{0} \delta\right)+ \\
& +\left[\varphi_{2}\left(\varepsilon_{0} \delta\right)-1\right] \ln (\delta)+\left[\varphi_{2}\left(\varepsilon_{0} \delta\right)-\varphi_{2}\left(\varepsilon_{0}\right)\right] \ln \left(\varepsilon_{0}\right), \\
\varphi_{1}(x)= & \frac{1+3 x+x^{2}}{2\left(1-x^{2}\right)^{\frac{1}{2}}(1+x)}, \quad \varphi_{2}(x)=\frac{2+2 x-3 x^{2}+2 x^{3}+2 x^{4}}{2\left(1-x^{2}\right)^{\frac{3}{2}}(1+x)}
\end{aligned}
$$

for nanotube [6], respectively. Since the linear approximation of the Gibbs-Thomson boundary conditions (Eqs. 4) is satisfactory only at $r_{\mathrm{i}}>3 \beta$ (as mentioned above), the lowest value of $\delta$ given by Eqs. 12 and 13 cannot be equal to zero. It should obey the following condition: $\delta>\varepsilon_{0}^{-1}\left[1+\left(r_{\mathrm{f}} / 3 \beta\right)^{q}\right]^{-\frac{1}{q}}$. Therefore, it is reasonable to define a collapse time as the time during which the magnitude of $\delta$ will decrease $e$ times, namely: $\tau_{\mathrm{c}}=\tau\left(\delta=e^{-1}\right)$. Hence, Eqs. 12 and 13 can be employed to find the collapse time only for the nano-object with geometrical parameters obeying the following condition: $\varepsilon_{0}>e\left[1+\left(r_{\mathrm{f}} / 3 \beta\right)^{q}\right]^{-\frac{1}{q}}$. It should be noted that $\varepsilon^{q}$ is the ratio of the number of virtual lattice sites within the void to the total number of lattice sites inside the whole of the nano-object, i.e.:

$$
\varepsilon^{q}=\frac{r_{\mathrm{i}}^{q}}{r_{\mathrm{e}}^{q}}=\frac{N_{\mathrm{V}}^{\text {void }}}{N+N_{\mathrm{V}}^{\text {shell }}+N_{\mathrm{V}}^{\text {void }}},
$$

where $N$ is the numbers of atoms and $N_{\mathrm{V}}^{\text {shell }}$ is the numbers of vacancies within the shell of the nano-objects respectively and $N_{\mathrm{V}}^{\mathrm{void}}$ is the number of vacancies in the void of the nano-objects.

\section{Kinetic Monte Carlo simulation}

$\mathrm{KMC}$ simulations of the shrinking kinetics by vacancies of the pure element hollow nano-objects were performed on f.c.c. lattices with lattice parameter $a$. The initial hollow nanosphere and nanotube had an inner radius $r_{\mathrm{i} 0}=7 a$ and external radius $r_{\mathrm{e} 0}=17 a\left(\varepsilon_{0} \approx 0.41\right)$, and consisted of 76750 and 51272 atoms, respectively. The nanotube had a linear size $H=17 a$ and its axis was oriented along the [001] direction ( $z-$ direction). Therefore, periodic boundary conditions along the $z$-direction were imposed for the nanotube. No 
initial vacancies were introduced inside the shells itself of the nano-objects. We consider nearest neighbour pair-interactions. The reduced pair-interaction energies $\phi / k T=-1.5$ were chosen using the expression [5]:

$$
-\frac{6 \phi}{k T} \approx \frac{1}{1-2 c_{\mathrm{V}}^{\mathrm{eq}}} \ln \left(\frac{1-c_{\mathrm{V}}^{\mathrm{eq}}}{c_{\mathrm{V}}^{\mathrm{eq}}}\right)
$$

to provide on the one hand reasonable calculation speed and, on the other, an equilibrium vacancy concentration $c_{\mathrm{V}}^{\mathrm{eq}} \sim 1.24 \times 10^{-4}$ close to the vacancy concentration at the melting temperature $T_{\mathrm{m}}$. We chose the Metropolis algorithm for its transparency and to provide a clear relationship between KMC steps per atom (KMCSA) $n$ and real time $t$. For this case, the relation evidently is $t=n / \Gamma_{0}$, where $\Gamma_{0}=12 v_{0}$ and $v_{0}$ is the effective attempt frequency. Then it follows immediately that $D_{\mathrm{V}} t=a^{2} n / 12$ (for this model $D_{\mathrm{V}}=a^{2} v_{0}$ ). Thus, if Eqs. 12 and 13 are valid then using them for the $\tau$ calculation on the basis of $N_{\mathrm{V}}^{\text {shell }}$ and $N_{\mathrm{V}}^{\text {void }}$ determined during KMC simulations and, next, determination of $\varepsilon$ (or $\delta$ ) with aid of Eq. 15, it follows from Eq. 10 that we should get a linear dependence of $\tau$ versus $n$.

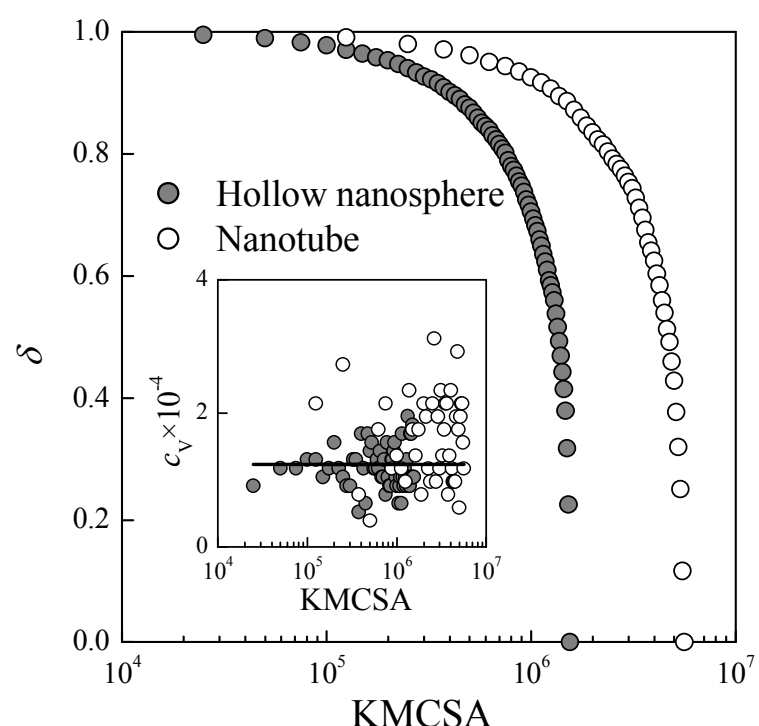

Fig. 1. KMC simulation of the shrinking kinetics of the f.c.c. nano-objects. The normalized size parameter is $\delta=\varepsilon / \varepsilon_{0}$. The inset shows instant vacancy concentrations in a shell in simulation (points) and average one predicted by Eq. 16 (line).

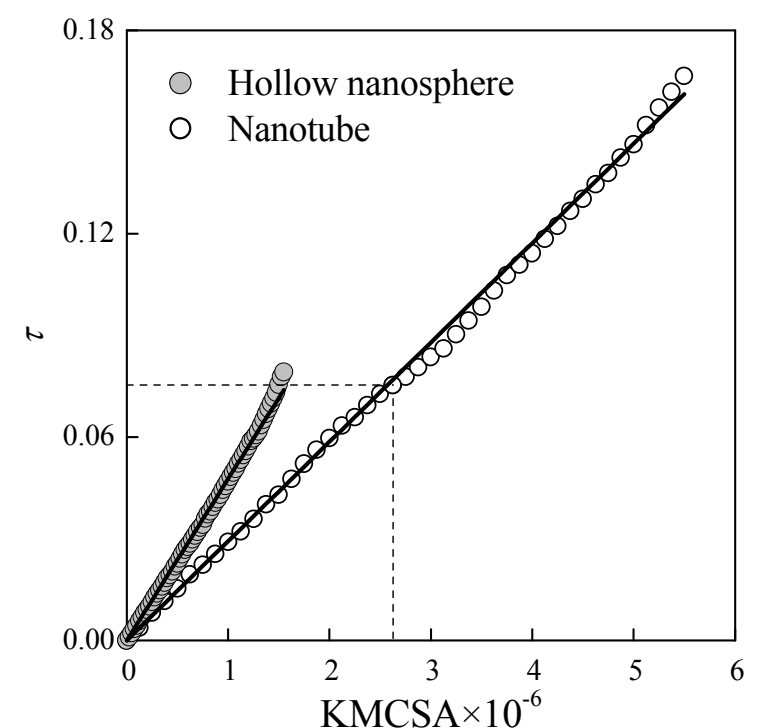

Fig. 2. Test of Eqs. 12 and 13 on the basis of the results of KMC simulation shrinking kinetics of a pure element f.c.c. nano-objects (see Fig. 1). The dashed lines indicate the point with $r_{\mathrm{i}} \approx 3 \beta_{\mathrm{c}}$ for the nanotube.

In Fig. 1, the changes of the normalized size parameter $\delta$ are shown during KMC simulation of the shrinking kinetics of the f.c.c. hollow nano-objects. For the nanotube, as can be seen from Fig. 2, $\tau$ calculated from this data according to Eq. 13 depends linearly from $n$ at least until condition $r_{\mathrm{i}}>3 \beta_{\mathrm{c}}$ is valid. Selfconsistent linear fitting of the data allows the determination of the parameter $\beta_{\mathrm{c}} \approx 1.764 a$ (see Eq. 10) only using the points satisfying the condition $r_{\mathrm{i}}>3 \beta_{\mathrm{c}}$ (see Fig. 2, the point with $r_{\mathrm{i}} \approx 3 \beta_{\mathrm{c}}$ indicated by dashed lines). However, the results of KMC simulation of shrinkage of a nanotube at range $r_{\mathrm{i}}<3 \beta_{\mathrm{c}}$ are also described satisfactorily by Eq. 13 (Fig. 2). Unfortunately, it is extremely time-consuming to perform KMC simulation of a hollow nanosphere model at the range $r_{\mathrm{i}}>3 \beta_{\mathrm{s}}$ (since $\beta_{\mathrm{s}}$ is two times larger $\beta_{\mathrm{c}}$ ) in order to verify directly and quantitatively Eq. 12 . Nonetheless, first we estimated $\beta_{\mathrm{s}}=2 \beta_{\mathrm{c}} \approx 3.528 a$, and then using the results of KMC simulation of the hollow nanosphere (Fig. 1) calculated $\tau$ according to Eq. 12 and approximated its dependence from $n$ by linear function (Fig. 2). Despite the fact that for the investigated hollow nanosphere already at the beginning $r_{\mathrm{i} 0}<3 \beta_{\mathrm{s}}$, the kinetics of its shrinkage is described reasonably by Eq. 12. Thus, the results of the KMC simulation confirm the phenomenological analysis described above and moreover, show the possibility of the application of Eqs. 12 and 13 even in range $r_{\mathrm{i}}<3 \beta$. 


\section{Molecular Dynamics Simulation}

The initial hollow nanosphere and nanotube with inner $r_{\mathrm{i} 0} \approx 28 \AA$ and external $r_{\mathrm{e} 0} \approx 65 \AA$ radii were cut from a perfect bulk f.c.c. lattice with a Pd lattice parameter $a=3.89 \AA$ and consisting of 76657 and 51068 atoms in the nanoshell, respectively. The nanotube had a linear size $H=66.13 \AA$ and its axis was oriented along the [001] direction ( $z$-direction), where periodic boundary conditions were imposed. Then the static relaxation procedure was applied. Interatomic interactions were described using a set of EAM functions, which were fitted to Pd bulk equilibrium solid properties and provided a good description of the liquid state [8]. Following this, MD procedure of isothermal annealing was performed. The MD procedure consisted of a numerical integration of the equations of atomic motion according to the Verlet algorithm with a time step $\Delta t=1.5 \mathrm{fs}$. During the simulation the momentum and the angular momentum of the whole system were conserved at zero values. Periodically, without an effect to the continuity of the annealing procedure, the system was transferred to a state at $T=0 \mathrm{~K}$ where atoms occupied equilibrium positions in local potential minima by making use of the static relaxation method. After this, the atomic movements occurring in the model were analyzed.
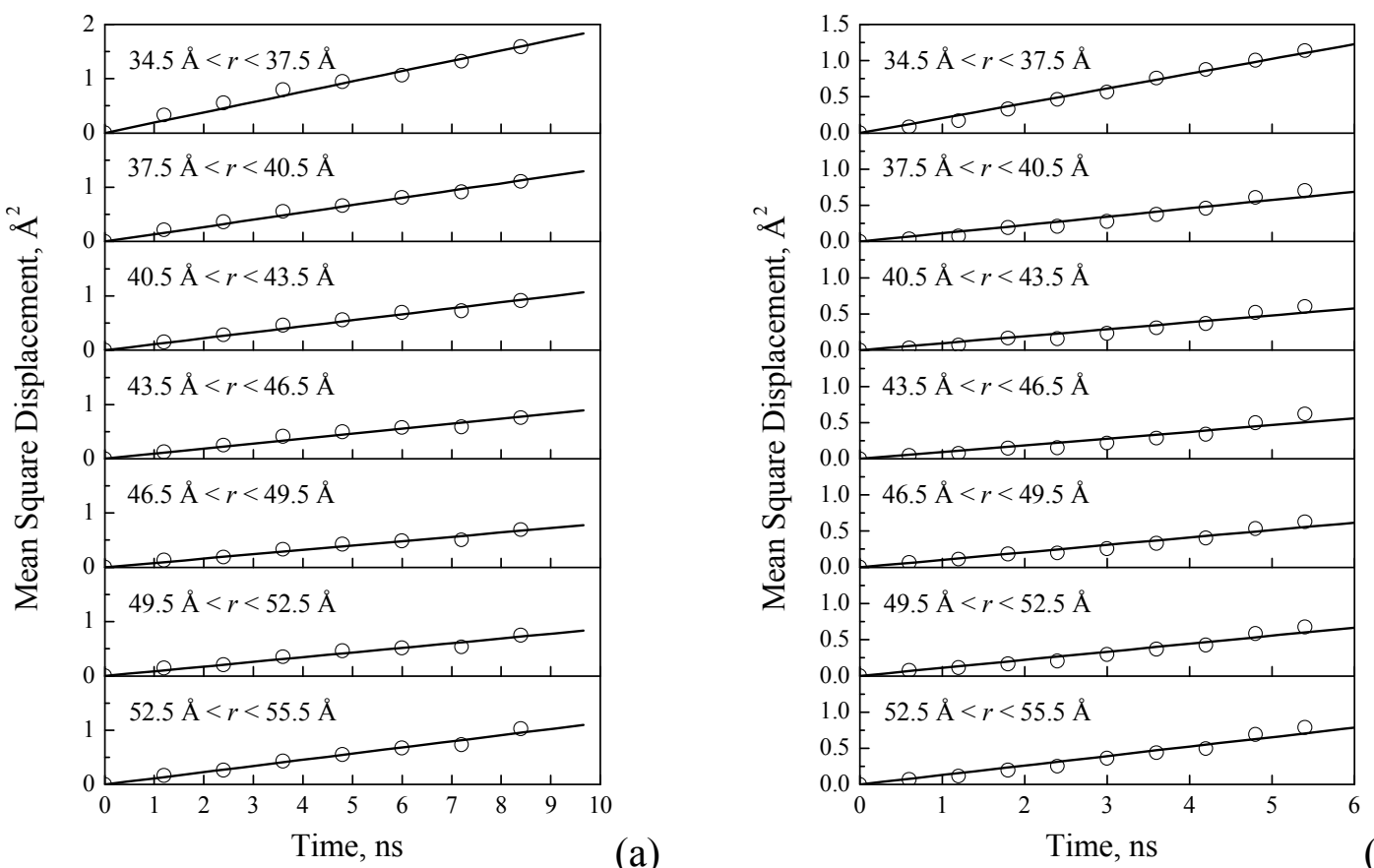

(a)

Fig. 3. Mean square displacement of Pd atoms by the vacancy mechanism inside the shells of (a) the hollow nanosphere and (b) nanotube (in the directions perpendicular to the axis of nanotube).
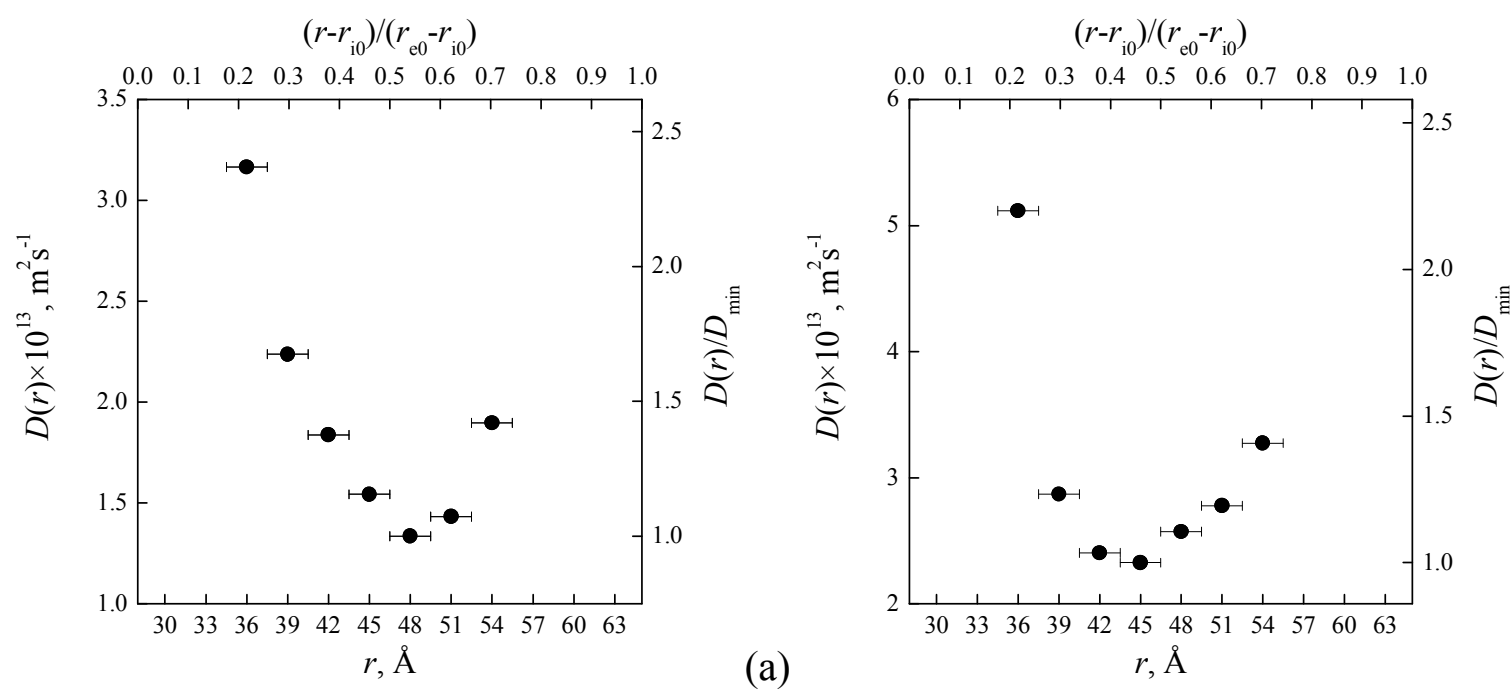

Fig.4. Diffusion coefficient by the vacancy mechanism inside the shells of (a) the hollow nanosphere and (b) nanotube (in the directions perpendicular to the axis of nanotube) versus radius. 
It was established that the investigated Pd hollow nano-object MD models melt at $1500 \mathrm{~K}$ [9] i.e. 18\% less than the melting temperature of a bulk Pd crystal $1825 \mathrm{~K}$. This difference can be associated not only with the interatomic potential, but also with a real decrease in melting temperature of a hollow nano-object in comparison with the bulk crystal. Therefore, our MD study of diffusion in Pd hollow nano-objects was carried out just below the melting temperature at $1450 \mathrm{~K}$. Before the diffusion calculations a vacancy concentration was permitted to approach equilibrium (this took the first $\sim 3 \mathrm{~ns}$ of our experiment, during this time the average diffusion length of a vacancy was more than $40 \AA$, which is larger than the nanoshells thickness $\sim 37 \AA$, for details see [9]). Then, for different distance intervals in nanoshells we have calculated the mean square displacement (MSD) only for those Pd atoms that during the time of experiment were in the same distance interval (see Fig. 3). It should be noted that outside the intervals indicated in Fig. 3 the MSD is much larger. Therefore, the average diffusion length was confined to less than $3 \AA$. Thus, outside these distance it was impossible to determine correctly the diffusion coefficient by the vacancy mechanism using this procedure. The diffusion coefficient of Pd atoms in different distance intervals in the nanoshells was calculated from the Einstein equation. It can be seen in Fig. 4 that the diffusion coefficient in the Pd hollow nano-object increases from the middle part of the nanoshell towards both inner and outer surfaces. Since the atomic diffusion coefficient is proportional to the vacancy concentration (assuming vacancy diffusion mechanism) [7], these findings suggest that the vacancy concentration has a minimum in the middle part of the nanoshell and increases towards the inner as well as external surfaces. This leads to a reasonable assumption: the hollow nano-objects are in a state corresponding to the local minimum of free energy. Therefore, growing of the vacancy concentration in the shells will result in the increasing of the free energy of such systems, namely to the increasing of the free energy part, which is responsible for the volume of the nanoshells. Thus, some free energy barrier will obstruct the transition of the hollow nano-objects by vacancy mechanism to the state corresponding to the global minimum of free energy (shrinkage to solid nano-objects). Hence, we have $c_{\mathrm{V}}^{0}>c_{\mathrm{V}}^{\text {eq }}$. As a result, in the phenomenological analysis the substitution of $c_{\mathrm{V}}^{\text {eq }}$ for $c_{\mathrm{V}}^{0}$ in Eq. 3 seems to be incorrect and KMC simulation cannot describe surface and subsurface relaxation and correctly $c_{\mathrm{V}}^{0}$.

\section{Summary}

We have analytically described the shrinkage by the vacancy mechanism of a pure element hollow nanosphere and nanotube. An exact solution of the kinetic equation has been obtained in quasi steady-state using Gibbs-Thomson boundary conditions at the linear approximation. The collapse time as a function of the geometrical sizes of the hollow nano-objects has been determined. Kinetic Monte Carlo simulation of the shrinkage of these nano-objects was performed: it confirmed the main results of the phenomenological analysis. In addition, molecular dynamics simulation in combination with the embedded atom method has been employed to investigate diffusion by the vacancy mechanism in a Pd hollow nanosphere and nanotube. We found that the atomic diffusion coefficient in the Pd hollow nano-objects models increases, as the measurement area recedes from the middle part of the nanoshells toward inner as well as external surfaces. Since the atomic diffusion coefficient is proportional to vacancy concentration we can suppose that in reality a hollow nanosphere and nanotube will not shrink as readily by the vacancy mechanism as predicted by the phenomenological analysis and kinetic Monte Carlo simulation.

Acknowledgements. This research was supported by the Australian Research Council. One of us (E.V.L.) wishes to thank the University of Newcastle for the award of a Research Fellowship.

\section{References}

[1] Y. Sun, B. Mayers, Y. Xia: Adv. Mater. Vol. 15 (2003), p. 641.

[2] Y. Yin, R.M. Rioux, C.K. Erdonmez, et al.: Science Vol. 304 (2004), p. 711.

[3] K.N. Tu and U. Gösele: Appl. Phys. Lett. Vol. 86 (2005), p. 093111.

[4] A.M. Gusak, T.V. Zaporozhets, K.N. Tu and U. Gösele: Phil. Mag. Vol. 85 (2005), p. 4445.

[5] A.V. Evteev, E.V. Levchenko, I.V. Belova, G.E. Murch: Phil. Mag. Vol. 87 (2007), p. 3787.

[6] A.V. Evteev, E.V. Levchenko, I.V. Belova, G.E. Murch: Def. Diff. Forum Vol. 266 (2007), p. 39.

[7] H. Mehrer: Diffusion in Solids (Springer-Verlag, Germany 2007).

[8] S.M. Foiles: Phys. Rev. B Vol. 32 (1985), p. 3409.

[9] A.V. Evteev, E.V. Levchenko, I.V. Belova, G.E. Murch: Sol. St. Phen. Vol. 129 (2007), p. 125. 
Diffusion and Diffusional Phase Transformations in Alloys

doi:10.4028/3-908451-55-8

Theoretical Analysis and Atomistic Modelling of Diffusion and Stability of Pure Element Hollow Nanospheres and Nanotubes

doi:10.4028/3-908451-55-8.21 